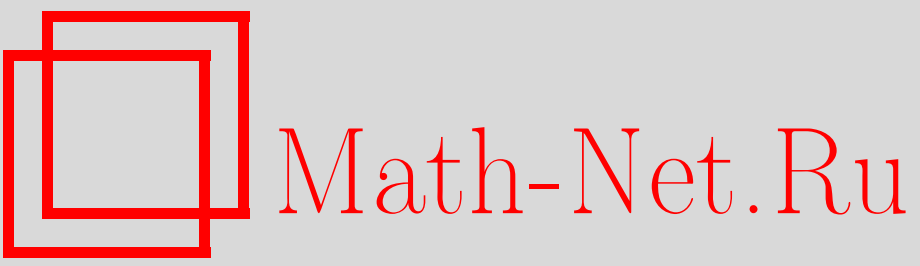

В. Е. Привальский, М. И. Фортус, О возможных причинах потепления климата, Теория вероятн. и ее примен., 2011, том 56, выпуск 2, 367-372

DOI: https://doi.org/10.4213/tvp4379

Использование Общероссийского математического портала Math-Net.Ru подразумевает, что вы прочитали и согласны с пользовательским соглашением

http://www . mathnet.ru/rus/agreement

Параметры загрузки:

IP : 3.85 .7 .115

26 апреля 2023 г., 16:42:00 
ТЕОРИЯ ВЕРОЯТНОСТЕЙ

Том 56

И ЕЕ ПРИМЕНЕНИЯ

Выпуск 2

2011

\title{
MISCELLANEA
}

(c) 2011 г. $\quad$ ПРИВАЛЬСКИЙ В. Е.*, ФОРТУС М. И.**

\section{О ВОЗМОЖНЫХ ПРИЧИНАХ ПОТЕПЛЕНИЯ КЛИМАТА}

\begin{abstract}
По данным инструментальных наблюдений за 1850-2009 гг. построена стационарная вероятностная модель колебаний температуры поверхности Земли и методом статистического моделирования показано, что явления, подобные происходящему сейчас потеплению, имеют возвратный период менее 500 лет, что немного для климата. Таким образом, для описания современных вариаций климата не обязательно привлекать внешние по отношению к климатической системе антропогенные воздействия.
\end{abstract}

Ключевые слова и фразы: статистическое моделирование, естественная изменчивость климата, климатические спектры.

1. Введение. За последние полвека средняя температура Земного шара повысилась примерно на $0.5^{\circ} \mathrm{C}$. Это потепление принято объяснять антропогенным ростом концентрации в атмосфере так называемых парниковых газов $\left(\mathrm{CO}_{2}\right.$, метан и др.); для компенсации антропогенных эффектов уже принимаются меры, которые фактически должны привести к изменению образа жизни человечества. Выводы о преимущественно антропогенных причинах потепления получены на основе экспериментов с численными моделями климата, проводящимися уже в течение многих лет. В важнейшей публикации, посвященной этим вопросам [3, с. 39], утверждается, в частности, что характеристики «наблюдаемого сейчас потепления воспроизводятся только моделями, которые содержат антропогенную вынуждающую силу... (см. также с. 665 и др.). Мы попытаемся показать, что это не обязательно так и происходящий сейчас рост температуры поверхности планеты может быть результатом низкочастотной изменчивости климата.

* Государственный океанографический институт им. Н. Н. Зубова, Кропоткинский пер., 6, 119034 Москва, Россия; e-mail: vprivalsky@oceanography.ru

${ }^{* *}$ Институт физики атмосферы им. А. М. Обухова РАН, Пыжевский пер., 3, 119017 Москва, Россия; e-mail: mfortus@yandex.ru 
2. Метод исследования и результаты. В статистической климатологии принято использовать понятия и методы теории стационарных случайных процессов. При этом наблюдаемые ряды значений различных климатических параметров рассматриваются как конечные реализации эргодического стационарного в широком смысле случайного процесса (разумеется, если это не противоречит здравому смыслу), а важнейшими их характеристиками являются средние значения, дисперсии, ковариационные функции, спектральные плотности (спектры) и другие «эмпирические» характеристики, вычисленные по этим реализациям с использованием формул математической статистики.

В течение последних десятилетий появляется все больше работ, содержащих эмпирические спектры реальных либо модельных рядов (в частности, полученных интегрированием больших гидродинамических моделей климата), а также рядов, полученных в результате палеореконструкций (см., например, [2] и ссылки, имеющиеся в этой работе). Эти ряды описывают поведение во времени разных элементов климатической системы (поверхностной температуры, функций тока, давления на уровне моря и др.) для отдельных пунктов, а также пространственно осредненных.

Такие спектры представляют распределение энергии для различных (но ограниченных!) временных масштабов - от десятков и сотен дней до тысяч и даже миллионов лет. Большинство спектров имеют заметный участок монотонного (в основном) роста с ростом временного масштаба (с уменьшением частоты). С приближением частоты к нулю спектры приближаются к горизонтальной прямой, так что соответствующий случайный процесс в области низких частот приближается к белому шуму. В данной работе для описания изменений центрированной средней годовой температуры поверхности Земли используется именно такая модель. Мы будем аппроксимировать колебания температуры $T_{t}$, где $t$ - дискретное время (годы), стационарным в широком смысле случайным процессом, наблюдаемым на выходе линейного фильтра следующего вида, на вход которого поступает белый шум $a_{t}$ с нулевым средним значением и дисперсией $\sigma_{a}^{2}$ :

$$
T_{t}=\sum_{j=1}^{p} \varphi_{j} T_{t-j}+a_{t} .
$$

Такие процессы носят название процессов авторегрессии порядка $p$, а коэффициенты авторегрессии $\varphi_{j}$ и дисперсия $\sigma_{a}^{2}$ подлежат оцениванию по имеющемуся временному ряду $T_{t}, t=1, \ldots, N$. Легко видеть, что спектральная плотность процесса, описываемого формулой (1), есть

$$
s(f)=2 \sigma_{a}^{2}\left|1-\sum_{j=1}^{p} \varphi_{j} e^{i 2 \pi j f \Delta t}\right|^{-2},
$$


где $\Delta t=1$ год, $f$ - частота (год ${ }^{-1}$ в интервале от 0 до $\left.1 /(2 \Delta t)\right)$. Получив оптимальные в некотором смысле оценки порядка модели, коэффициентов авторегрессии и дисперсии белого шума, можно замоделировать методом Монте-Карло любое число $N$ значений процесса (1) со спектральной плотностью (2) и, в частности, изучить распределение серий повышенной температуры $T_{t}$. Методы оценивания параметров временного ряда вида (1) описаны, в частности, в [1].

Ход наблюденной средней годовой температуры поверхности Земли показан на рис. 1 (см. [4]).

3. Результаты и выводы. Оценивание параметров модели (1) по ряду наблюдений с 1850 г. по 2009 г. приводит к следующему результату:

- порядок авторегрессии: $p=4$,

- оценки коэффициентов авторегрессии (и их среднеквадратические отклонения):

$$
\begin{aligned}
& \varphi_{1}=0.630 \pm 0.080, \\
& \varphi_{2}=-0.021 \pm 0.095, \\
& \varphi_{3}=0.100 \pm 0.095, \\
& \varphi_{4}=0.237 \pm 0.080, \\
& - \text { дисперсия белого шума: } \sigma_{a}^{2}=0.0128\left({ }^{\circ} \mathrm{C}\right)^{2}\left[\sigma_{a}=0.113^{\circ} \mathrm{C}\right],
\end{aligned}
$$

а дисперсия значений самого наблюденного ряда равна $0.0672\left({ }^{\circ} \mathrm{C}\right)^{2}$.

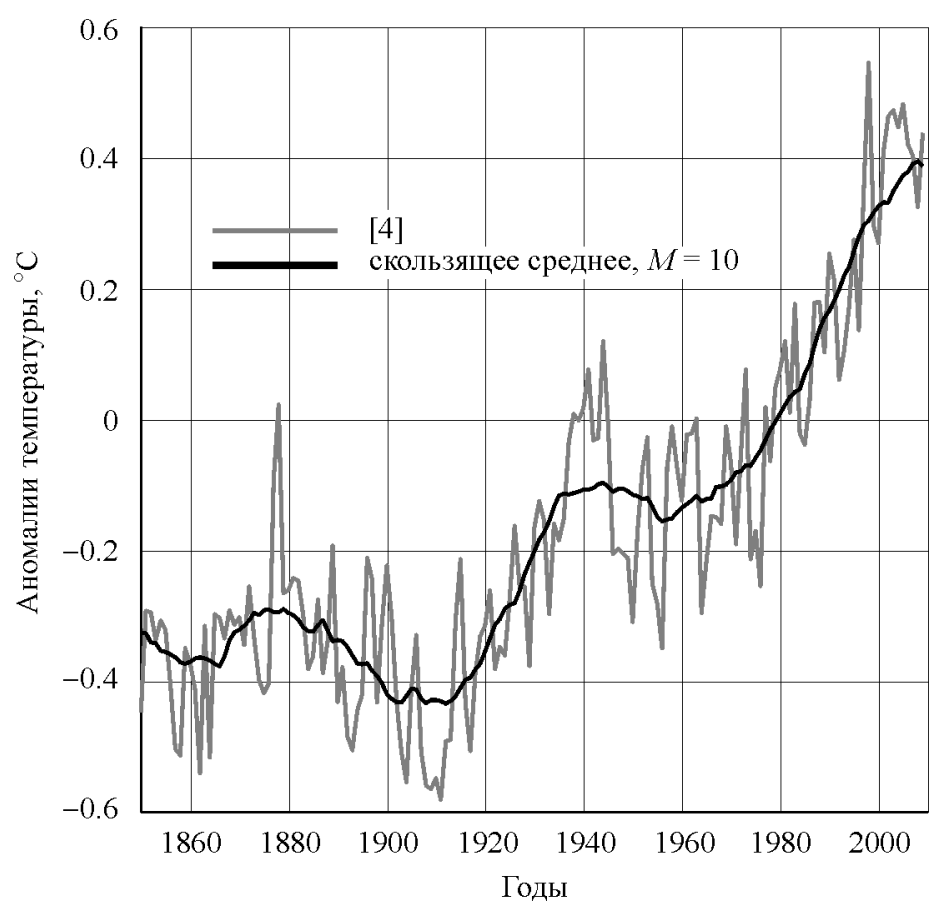

Рис. 1. Средняя годовая температура поверхности Земли, 1850-2009. 
Спектральная плотность процесса, отвечающего уравнению (1) с перечисленными выше параметрами, показана сплошной линией на рис. 2.

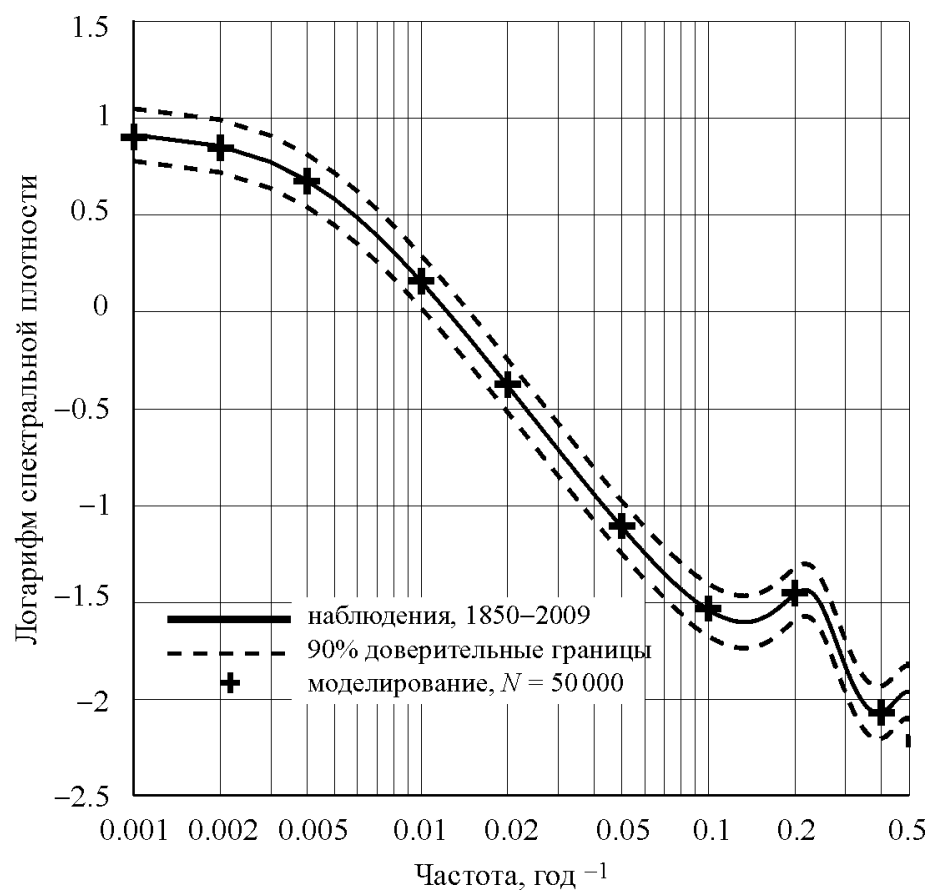

Рис. 2. Спектральная плотность наблюденной и моделированной температуры.

Зная параметры, можно моделировать реализации произвольной длины, которые будут иметь в точности те же статистические свойства, что и наблюденный ряд. Спектр такого модельного ряда длиной $N=50000$ также показан на рис. 2 , и, как видно, он точно совпадает с оценкой спектра ряда наблюдений.

При желании можно начинать моделирование с четырех первых и четырех последних значений наблюденной температуры, полностью включив таким образом наблюденный ряд в результаты моделирования. Соответствующий пример показан на рис. 3. Как видно, ряд наблюдений выглядит вполне естественно в последовательности модельных значений, представляющих собой возможный ход температуры поверхности Земли за 1000 лет.

Мы замоделировали 200000 значений температуры и проанализировали эти данные, чтобы посмотреть, как часто в нем встречаются серии, похожие на то, что наблюдается сейчас. Мы искали интервалы времени длиной от 35 до 65 лет, в течение которых значения модельной температуры, пропущенные через низкочастотный фильтр (см. рис. 1), возрастали почти монотонно примерно на $0.5^{\circ} \mathrm{C}$. Таких случаев за 200000 лет оказалось 410, откуда следует, что возвратный период серий подобного рода составляет менее 500 лет (точнее, 489 лет). Иными словами, 
случаи роста температуры на примерно $0.5^{\circ} \mathrm{C}$ за несколько десятилетий не представляют собой исключения в модельном климате: они занимают чуть более $10 \%$ времени и, стало быть, в любой данный момент температура поверхности Земли может проходить такую серию с вероятностью около 0.1, что не так мало. Этот вывод получен без привлечения внешней вынуждающей силы.

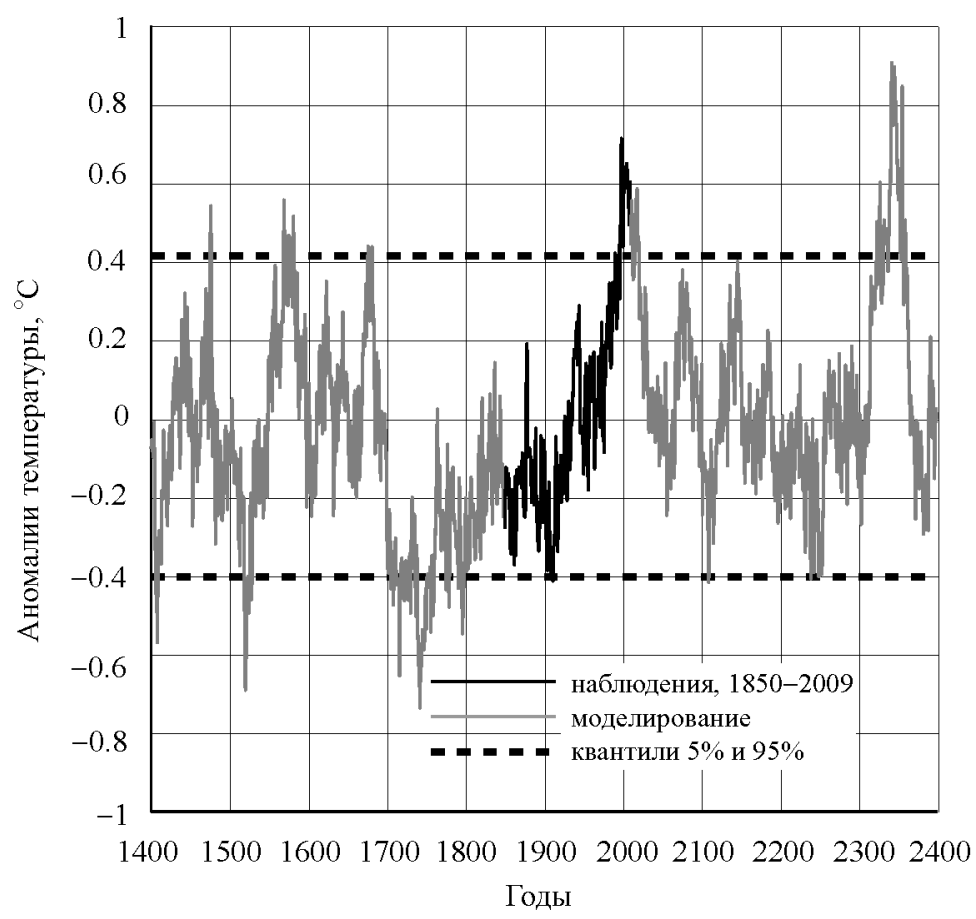

Рис. 3. Пример моделирования глобальной температуры.

Появление довольно длительных периодов потепления может быть связано с тем, что температурный спектр (как и многие климатические спектры) растет с убыванием частоты, из-за чего основная часть энергии сосредоточена в интервале временных масштабов, значительно превышающих длину интервала современного потепления. Мощная низкочастотная компонента ряда, если она в фазе роста, может в течение достаточно большого временного промежутка определять поведение ряда, так что будет казаться, что ряд содержит детерминированный тренд.

\section{СПИСОК ЛИТЕРАТУРЫ}

1. Прохоров Ю. В. (ред.). Вероятность и математическая статистика. М.: Большая Российская Энциклопедия, 1999, 910 с.

2. Фортус М. И. Определение доверительных интервалов при анализе климатических рядов. - Изв. РАН, ФАО, 2010, т. 46, № 5, с. 608-619. 
3. IPCC. Climate Change 2007. The Physical Science Basis. Contribution of Working Group I to the Fourth Assessment Report of the Intergovernmental Panel on Climate Change. Ed. by S. Solomon et al. Cambridge: Cambridge Univ. Press, 2007, 996 p.

4. Jones P.D., New M., Parker D. E., Martin S., Rigor I. G. Surface air temperature and its changes over the last 150 years. - Rev. Geophys., 1999, v. 37, p. 173-199; see also http://www.cru.uea.ac.uk/cru/data/temperature/.

Поступила в редакцию 